\title{
Turbulent transport in TCV plasmas with positive and negative triangularity
}

\author{
G. Merlo, ${ }^{1}$ M. Fontana, ${ }^{2}$ S. Coda,${ }^{2}$ D. Hatch,${ }^{3}$ S. Janhunen, ${ }^{1}$ L. Porte,${ }^{2}$ and F. Jenko ${ }^{1,4}$ \\ ${ }^{1}$ Oden Institute for Computational Engineering and Sciences, \\ The University of Texas at Austin, Austin, TX 78712. USA \\ ${ }^{2}$ Ecole Polytechnique Fédérale de Lausanne (EPFL), \\ Swiss Plasma Center (SPC), CH-1015 Lausanne, Switzerland \\ ${ }^{3}$ Institute for Fusion Studies, The University of Texas at Austin, Austin, TX 78712. USA \\ ${ }^{4}$ Max-Planck-Institut für Plasmaphysik, \\ Boltzmannstr. 2, D-85748 Garching, Germany
}

\begin{abstract}
Local gyrokinetic simulations with the GENE code are used to investigate the turbulent transport for different levels of NBI heating power in TCV plasmas with both positive and negative edge triangularity. The sensitivity of the heat fluxes with respect to the main plasma parameters, including background gradients, impurity content and electron temperature, is systematically studied. The experimentally measured transport levels are recovered with variations of profiles compatible with experimental error bars. When considering experimental conditions, TEMs are the dominant instabilities for all heating powers and both types of shapes, whereas ITGs are not found. A numerical experiment, considering plasma profiles that strongly destabilize ITG modes, is thus performed. Negative triangularity is found to reduce the transport level also in this regime.
\end{abstract}




\section{INTRODUCTION}

Negative triangularity has regained interest thanks to new experimental data confirming its beneficial influence reducing the turbulent transport in magnetic confinement fusion devices. New and more detailed TCV measurements have been collected, showing that $\delta<0$ induces a reduction of transport and of fluctuations, of both electron temperature and density [1, 2]. This confirms the original results by Camenen et al. presented in Ref. [3], where for plasmas with negative triangularity of the Last Closed Flux Surface (LCFS) $\delta_{\text {LCFS }}$, the same electron temperature and density profiles were obtained injecting half the heating power compared to their positive triangularity counterpart (in this particular case going from $\delta_{\mathrm{LCFS}}=0.4$ to -0.4$)$. This has been experimentally interpreted as a better electron energy confinement at all radii, and several theoretical and numerical investigations have been subsequently carried out in order to explain this observation [4-6]. A detailed understanding of the mechanism behind the effect of triangularity is however still missing.

Experimental investigations of negative triangularity have also been carried out recently in the DIII-D tokamak, where discharges with $\delta<0$ show H-mode-like confinement and high normalized beta with L-mode-like edge pressure profiles and no ELMs [7, 8]. These plasmas show the same global performance as a positive triangularity ELMing H-mode discharge with the same plasma current, elongation and area. A reduction of fluctuations and fluxes has been observed as well, confirming that negative triangularity is a viable candidate for reactor scenarios with high confinement.

In part because of these experimental findings, negative triangularity has been suggested as a possible solution for the design of future optimized tokamaks and fusion reactors, including DEMO $[9,10]$.

We recall that the original observation of an improvement of the energy confinement with $\delta<0$ in TCV reported by Camenen et al., was attributed to plasmas dominated by Trapped Electron Modes (TEMs) and the stabilizing effect was speculated to be due to a modification

of the toroidal precessional drift associated to $\delta_{\mathrm{LCFS}}<0$, in turn able to stabilize the TEMs [11]. These discharges were also characterized by a very large electron-to-ion temperature ratio $T_{e} / T_{i}$, exceeding three in the plasma core. The NBI heating system recently installed on TCV [12] represents a way of increasing the ion temperature towards values relevant for reactor operation. This is expected to potentially destabilize Ion Temperature Gradient 
(ITG) modes (as well as Electron Temperature Gradient (ETG) modes), thus allowing one to investigate whether or not the beneficial effect of negative $\delta$ persists in different turbulent regimes.

A numerical investigation of the effect of triangularity under these conditions is therefore desirable. Being based on more recent and accurate experimental data, this work can thus be considered complementary to the existing investigations available in literature. We will show that, for the TCV NBI heated cases considered here, one can recover the experimental transport level with local simulations by varying the profiles within the experimental error bars. Matching the correct flux level turns out to be simpler task for $\delta_{\text {LCFS }}>0$. One can obtain the same final outcome for the negative triangularity plasmas where, however, a reduction of the electron temperature, still compatible with experimental uncertainties, is also required. We already remark that despite a specific experimental effort put into having all plasma parameters except triangularity as close as possible between the different discharges, in fact one observes minor but non-negligible variations in all relevant parameters. Nonetheless, ITG modes have not been found as the leading instability for any of the plasmas considered here. Thus, in order to explore what would happen in that regime, an ad-hoc investigation has been carried out and will be discussed.

The remainder of this paper is organized as follows. A description of the shots is provided in Section II while a description of the simulation tool is given in Section III. Linear results obtained considering the experimental TCV plasmas are presented in Sec. IV whereas corresponding nonlinear ones are discussed in Sec. V. A dedicated investigation of the effect of negative $\delta$ on ITG dominated scenarios is presented in Section VI. Finally, conclusions are

drawn in Section VII. A brief discussion of the experimental profile fittings and associated error bars is given in Appendix A while linear sensitivity results are summarized in Appendix B.

\section{OVERVIEW OF THE EXPERIMENTAL PLASMA CONDITIONS}

We focus our attention on modeling the two TCV discharges \#60797 and \#58499, which are two L-mode plasmas with respectively $\delta_{\mathrm{LCFS}} \simeq 0.4$ and $\delta_{\mathrm{LCFS}} \simeq-0.4$. These discharges have been experimentally designed in order to study the impact of neutral beam injection (NBI) on the stabilizing effect of negative triangularity. A detailed description of the exper- 
imental set-up can be found in $[13,14]$.

The nominal physical plasma parameters characterizing the considered plasma are listed in Table I (resp. Table II) for the positive (resp. negative) $\delta$ case. Experimental electron temperature and density profiles are obtained from Thomson scattering, while ion ones are inferred from Charge Exchange Spectroscopy measurements of the main impurity (carbon). The deuterium concentration is evaluated from quasi-neutrality while its temperature profile is considered the same as the carbon one. More details regarding fitting and error bar estimates are given in Appendix A. Temperature and density gradient lengths are defined according to the following relation:

$$
a / L_{T, n}=-d \log (T, n) / d \rho_{t o r} .
$$

with $a=\sqrt{\Phi_{\mathrm{LCFS}} / \pi B_{0}}, \Phi$ being the toroidal flux. Here $i \rho_{\text {tor }}$ is the radial coordinate based on the toroidal flux $\Phi, \dot{i} \rho_{\text {tor }}=\sqrt{\Phi / \Phi_{\mathrm{LCFS}}}$. The experimental value of $\beta_{e}=2 \mu_{0} p_{e} / B_{0}^{2}$ is used; $p_{e}$ is the local electron pressure. Finite collisionality is accounted for, retaining inter- and intra-species collisions. All collision frequencies are consistently derived from the value of $\nu_{e i}$ and local values of temperature and density. In particular, $\nu_{e i}=\sum_{i} 3 \sqrt{\pi} / 4 \tau_{e, i}$, where $\tau_{e, i}$ is the electron collision time of the $i$-th ion species, $\tau_{e, i}=3(2 \pi)^{3 / 2} \epsilon_{0}^{2} T_{e}^{3 / 2} m_{e}^{1 / 2} / n_{i} Z_{i}^{2} e^{2} \log \Lambda, m_{e}$ is the electron mass and $\log \Lambda$ is the Coulomb logarithm. In Tables I and II we also list the values of the effective collisionality $\nu_{\text {eff }}=\nu_{e i} / \omega_{D e}$, which compares the electron-ion collision frequency to the electron curvature drift frequency, obtained from the approximation $\nu_{\text {eff }}=$ $0.1 R n_{e} Z_{\text {eff }} / T_{e}^{2}$ provided in Ref. [15].

We have considered three different plasma conditions for the $\delta>0$ case (shot \#60797) and two for the $\delta<0$ one (shot \#58499) in order to allow for a comparison between scenarios as similar as possible except for $\delta$ variations. We nonetheless remark that even though the experiments were designed to isolate any effect of triangularity, minor variations are in fact found in all major plasma parameters. Ohmic conditions will be referred to in the following as Phase I. Phase II are plasmas in which the same NBI power $(300 \mathrm{~kW})$ is injected, resulting however in different profiles for the two shapes. We therefore consider also Phase III for the $\delta>0$ scenario, where $1 \mathrm{MW}$ is injected in order to obtain profile matching as close as possible Phase II of $\delta<0$. All simulations are carried out at the position $\rho_{\text {tor }}=0.685$, which is where ECE measurements are available and triangularity, which has a finite radial penetration depth, is still large $(\approx \pm 0.2)$. The validity of the local approximation will be 


\begin{tabular}{|c|c|c|c|}
\hline & Phase I (t=0.61 s) & Phase II $(\mathrm{t}=1.10 \mathrm{~s})$ & Phase III $(\mathrm{t}=1.57 \mathrm{~s})$ \\
\hline$q$ & 1.68 & 1.68 & 1.67 \\
\hline$\hat{s}$ & 1.53 & 1.55 & 1.61 \\
\hline$T_{i} / T_{e}$ & 1.24 & 1.13 & 1.51 \\
\hline$T_{e}[\mathrm{keV}]$ & 0.23 & 0.33 & 0.31 \\
\hline$n_{e}\left[10^{19} 1 / m^{3}\right]$ & 2.20 & 1.93 & 1.93 \\
\hline$n_{c} / n_{i}$ & $0.65 \times 10^{-2}$ & $0.52 \times 10^{-2}$ & $0.76 \times 10^{-2}$ \\
\hline$a / L n_{e}$ & 2.56 & 2.13 & 1.43 \\
\hline$a / L n_{i}$ & 2.62 & 2.20 & 1.49 \\
\hline$a / L n_{c}$ & 1.21 & 0.66 & -0.66 \\
\hline$a / L_{T_{e}}$ & 4.76 & 2.70 & 3.90 \\
\hline$a / L_{T_{i}}$ & 1.79 & 1.57 & 1.71 \\
\hline$\nu_{e i}\left[c_{s} / a\right]$ & 1.12 & 0.51 & 0.55 \\
\hline$\nu_{\mathrm{eff}}$ & 4.20 & 1.97 & 2.03 \\
\hline$\beta_{e}$ & $0.09 \times 10^{-2}$ & $0.12 \times 10^{-2}$ & $0.11 \times 10^{-2}$ \\
\hline $\mathrm{NBI}[\mathrm{kW}]$ & 0 & 300 & 900 \\
\hline
\end{tabular}

TABLE I. Plasma parameters used for carrying out local simulation for TCV shot \#60797 $(\delta>0)$. All parameters are evaluated at $\rho_{\text {tor }}=0.685$.

discussed in the following. A magnetic reconstruction of the experimental equilibria has been carried out with the LIUQE code [16] in order to determine the location of the LCFS based on magnetic measurements. Such equilibria have then been further processed with CHEASE [17] in order to obtain the metric coefficients in straight-field-line coordinates as needed in GENE.

\section{NUMERICAL SIMULATION TOOL}

The gyrokinetic simulations shown here have been carried out using the local (flux-tube) version of the GENE code [18]. GENE is an Eulerian code developed for linear and non-linear simulations of plasma microturbulence. It allows one to consider fully kinetic multi-species dynamics, including electrostatic and electromagnetic fluctuations, and intra- and inter- 


\begin{tabular}{|c|c|c|}
\hline & Phase I $(\mathrm{t}=0.75 \mathrm{~s})$ & Phase II $(\mathrm{t}=1.10 \mathrm{~s})$ \\
\hline$q$ & 1.79 & 1.79 \\
\hline$\hat{s}$ & 1.38 & 1.39 \\
\hline$T_{i} / T_{e}$ & 1.20 & 1.78 \\
\hline$T_{e}[\mathrm{keV}]$ & 0.33 & 0.45 \\
\hline$n_{e}\left[10^{19} 1 / m^{3}\right]$ & 1.76 & 2.01 \\
\hline$n_{c} / n_{i}$ & $0.14 \times 10^{-2}$ & $0.13 \times 10^{-2}$ \\
\hline$a / L n_{e}$ & 2.85 & 3.61 \\
\hline$a / L n_{i}$ & 2.82 & 3.62 \\
\hline$a / L n_{c}$ & 3.23 & 3.56 \\
\hline$a / L_{T_{e}}$ & 4.41 & 4.67 \\
\hline$a / L_{T_{i}}$ & 1.72 & 2.09 \\
\hline$\nu_{e i}\left[c_{s} / a\right]$ & 0.55 & 0.53 \\
\hline$\nu_{\mathrm{eff}}$ & 1.93 & 1.07 \\
\hline$\beta_{e}$ & $0.11 \times 10^{-2}$ & $0.14 \times 10^{-2}$ \\
\hline $\mathrm{NBI}[\mathrm{kW}]$ & 0 & 300 \\
\hline
\end{tabular}

TABLE II. Same as Table I but listing parameters of the $\delta<0$ case, i.e. TCV shot \#58499.

species collisions (both pitch angle and energy scattering)[19].

GENE adopts a field aligned coordinate system $(x, y, z)$ to discretize the configuration space, while $\left(v_{\|}, \mu\right)$ are used as velocity variables. Here $x$ stands for the radial, $y$ for the binormal and $z$ is the straight-field line poloidal angle parametrizing the position along a given field line, and therefore is referred to as "parallel" direction. The variable $\mu=m v_{\perp}^{2} / 2 B$ represents the magnetic moment, while $v_{\|}$and $v_{\perp}$ the components of velocity respectively parallel and perpendicular to the magnetic field; $m$ is the mass of the particle and $B$ the local magnitude of the magnetic equilibrium field $\mathbf{B}$. In the flux-tube version of the code, a Fourier representation is used for both the $x$ and $y$ directions. To ensure numerical convergence of each simulation, different box sizes $L_{x} \times L_{y}$ have been used. Typical values are $L_{x} \sim L_{y} \sim$ $150 \rho_{s}$, considering $256 k_{x} \times 64 k_{y}$ modes. $48 n_{z}$ points have been used in the parallel direction. The limits for the velocity space grids of each $j$-species were set from -3 to 3 for $v_{\|} / v_{t h, j}$ and from 0 to 9 for $\mu B_{0} / T_{j}, v_{t h, j}=\sqrt{2 T_{j} / m_{j}}$ being the thermal velocity and $B_{0}$ 
the magnetic field on axis, using unless differently stated $48 \times 16$ points.

\section{LINEAR ANALYSIS}

\section{A. Positive triangularity plasma}

Linear simulations have been carried out considering the nominal value of the measured plasma profiles. Growth-rate $\gamma$ and real frequency $\omega_{r}$ of the most unstable mode are shown in Figure 1 for mode numbers $k_{y} \rho_{s}$ at the ion scale $\left(k_{y} \rho_{i} \sim 1\right)$. Growth rates for modes up to the electron scale $\left(k_{y} \rho_{e} \sim 1\right)$ are depicted in Figure 2 .
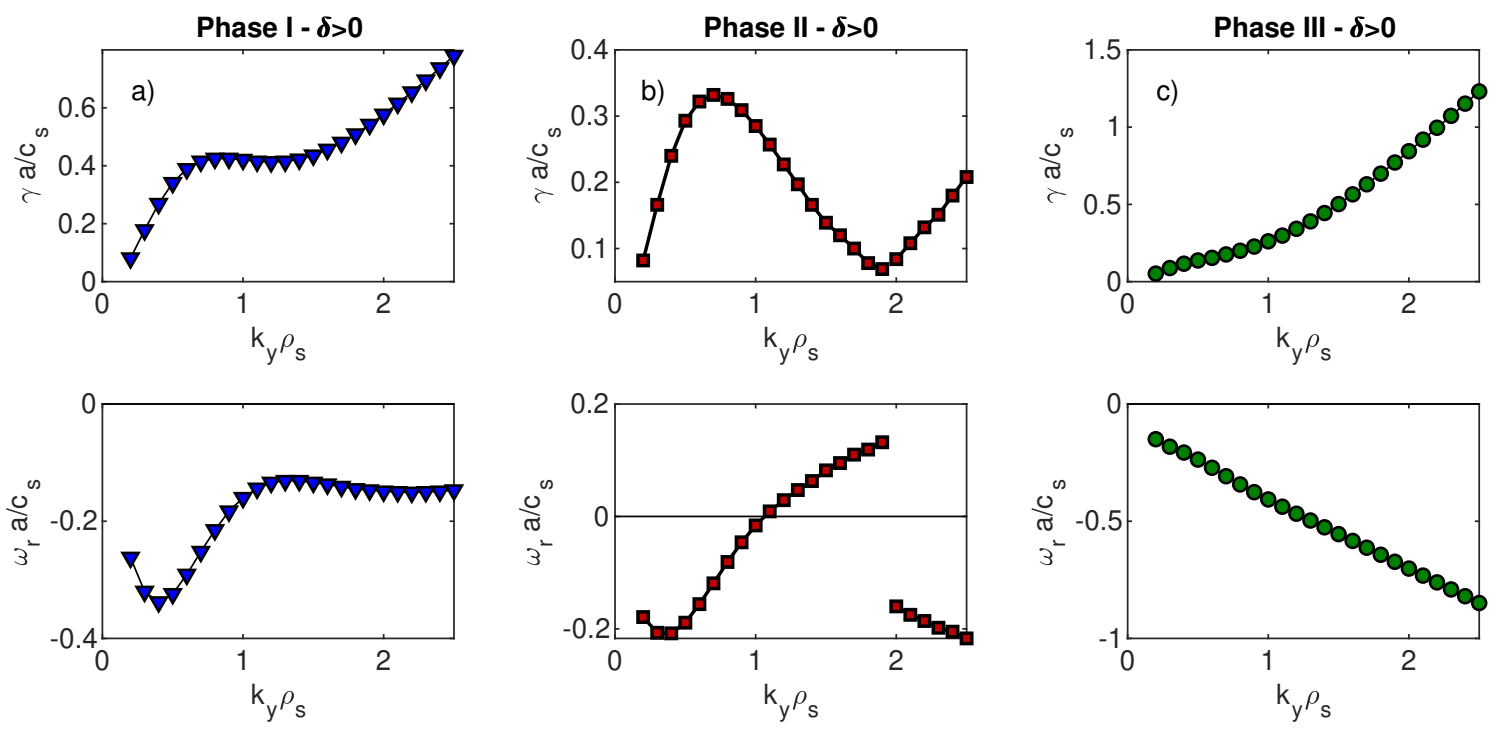

FIG. 1. Growth-rate $\gamma$ and real frequency $\omega_{r}$, in units of $a / c_{s}$ as function of the toroidal mode number $k_{y} \rho_{s}$. All cases characterized by $\delta>0$.

We observe that for all cases considered here the dominant instabilities are characterized by a negative frequency, which corresponds in the GENE conventions to a propagation in the electron diamagnetic direction. Based on this, and of the fact that unstable modes are found up to the electron scale, we interpret them as a mixture of TEM and ETG. It appears difficult to exactly distinguish between those two instabilities given that, with the exception of Phase II (see red curves in Fig. 1 and 2), we find a continuous transition from ion to electron scale modes. Comparing the different NBI phases, we observe that when the beam is switched on there is first a reduction of the growth rates (by approximately $30 \%$ 


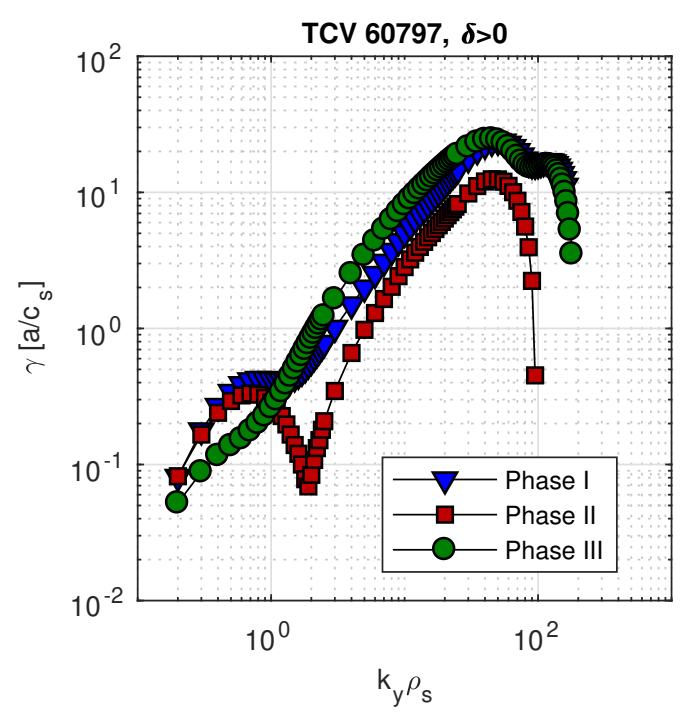

FIG. 2. Growth-rate $\gamma$ up to the electron scales as function of the toroidal mode number $k_{y} \rho_{s}$ $(\delta>0)$. Same parameters as of Figure 1.

at $k_{y} \rho_{s}=0.7$, the most unstable mode at the ion scale, and by almost a factor of two for the most unstable ETG mode) and a clear separation between TEM and ETG branch is observed around $k_{y} \rho_{s} \sim 1.9$. Then, with the beam at full power (green circles), ETG modes are destabilized and a continuous transition from TEM to ETG is again observed. We have investigated the sensitivity of microinstabilities to variations of the background profiles by varying $\pm 20 \%$ both electron temperature and density gradient scale-lengths. Simulation results are summarized in Appendix B; for the mode $k_{y} \rho_{s}=0.3$, where we expect the most important contribution to nonlinear fluxes, we observe an almost equal sensitivity of growth rates to both $a / L_{T_{e}}$ and $a / L_{n}$.

\section{B. Negative triangularity plasma}

The same linear analysis has been carried out for the $\delta<0$ plasma as shown in Figures 3 and 4. Again, we observe a combination of TEM and ETG, with modes equally sensitive to electron temperature and density gradient length variations.

Comparing the results obtained considering the two different discharges, we find that linear growth rates are larger in the negative triangularity case. While this can be somewhat surprising since plasmas with $\delta<0$ are supposed to be more stable than ones with $\delta>0$, we 

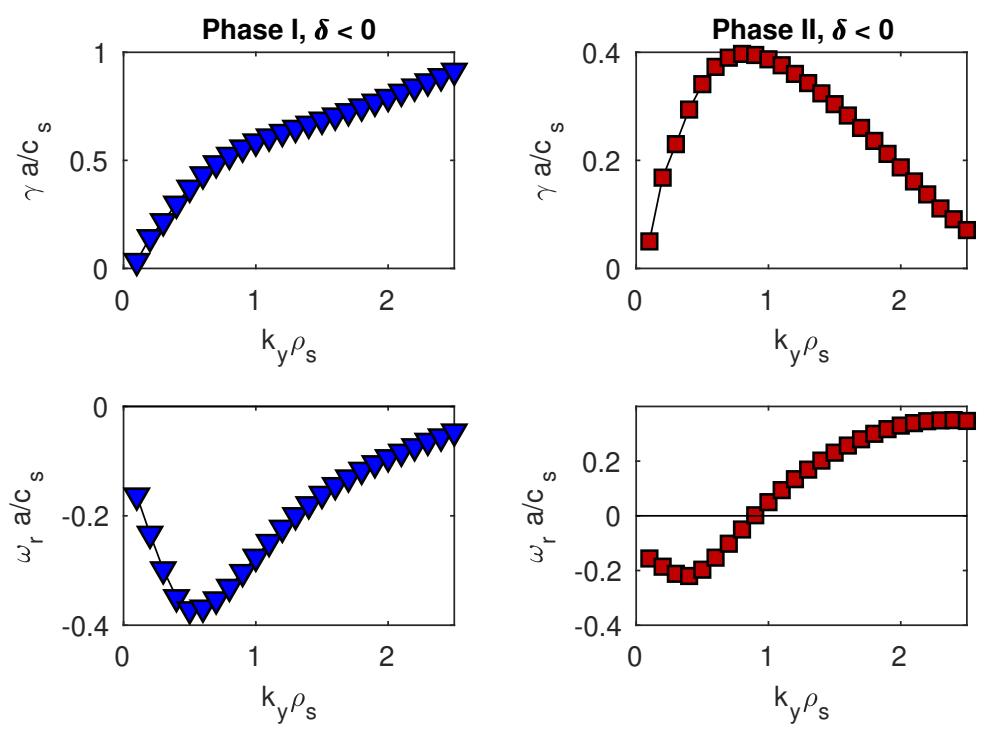

FIG. 3. Same as figure 1 but showing results for the negative triangularity case.

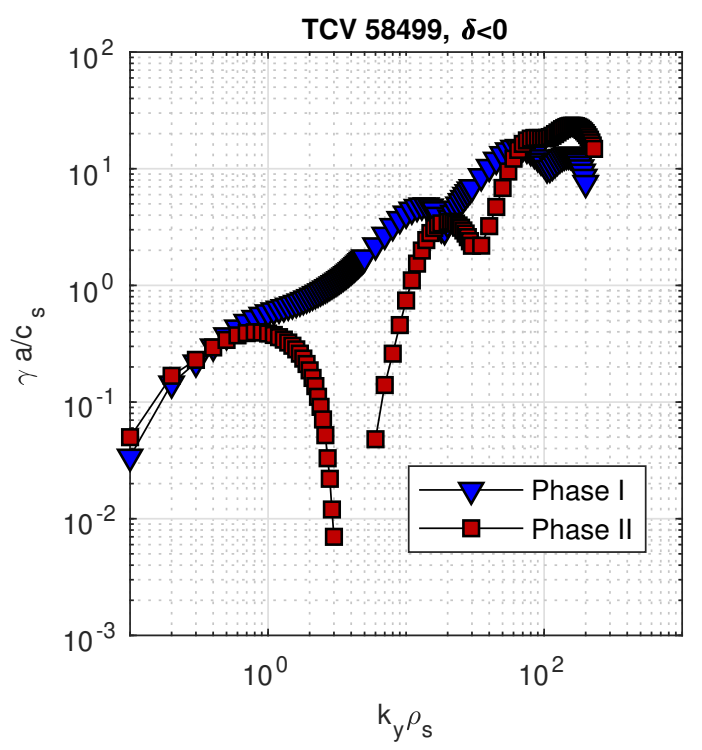

FIG. 4. Same as fig. 2 but showing results for the negative triangularity case.

remark that inputs gradients are larger by at least $10 \%$ for the $\delta<0$ case, thus potentially explaining the linear results. A simple way to assess the impact of negative $\delta$ (see e.g. Ref. [5]) would be to mix the experimental conditions, simulating e.g. the experimental profiles of $\delta>0$ but considering the magnetic geometry of $\delta<0$. Such an exercise should be done carefully, and only if supported by experimental evidence, as one would be carrying out simulations with inconsistent pressure profiles and MHD equilibria but one might also 
obtain results difficult to interpret. As an example, we compare in Figure 5 the linear growth
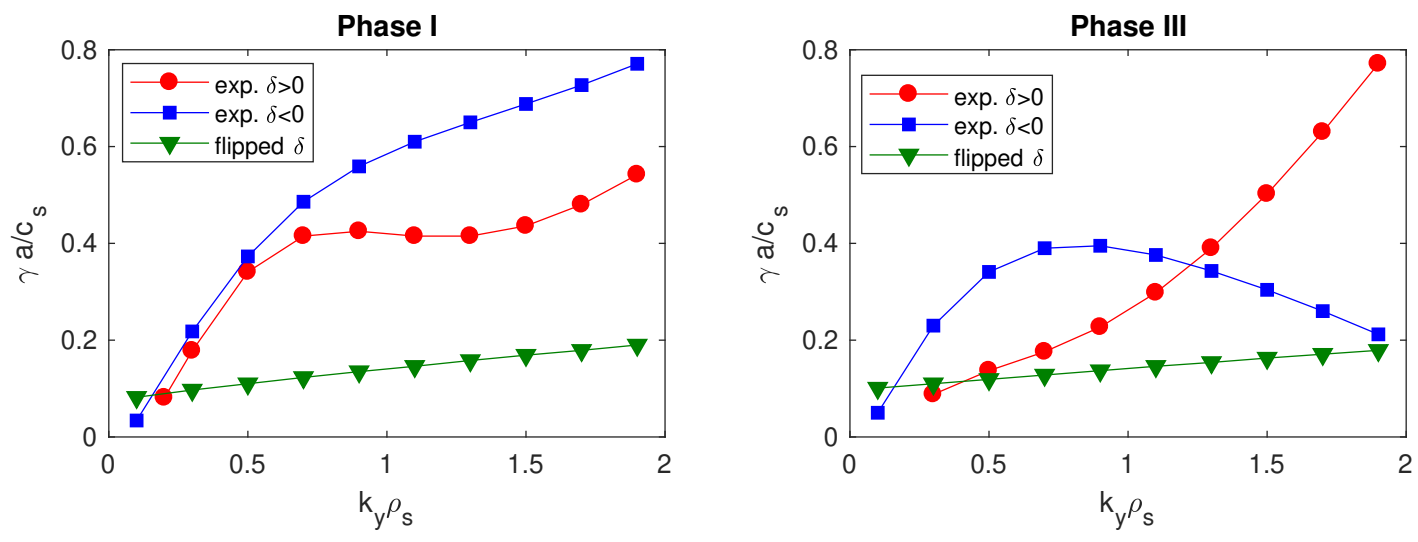

FIG. 5. Growth-rate $\gamma$ as function of the toroidal mode number $k_{y} \rho_{s}$ for (left) Phase I and (right) Phase III. Results for experimental condition corresponding to positive triangularity are shown with red circles, negative with blue squares and the ones obtained considering the equilibrium of $\delta<0$ but profiles of $\delta>0$ with green triangles.

rates obtained when simulating the experimental nominal conditions for both shapes (for Phases I and III) to the ones obtained when the negative triangularity equilibrium is used with temperature and density profiles of the its positive $\delta$ counterpart. While one observes a strong reduction of the growth rates for all $k_{y} \rho_{s}>0.5$, for the lowest $k_{y}$ modes (where we expect the nonlinear fluxes to peak) growth rates obtained with negative triangularity are lower than the ones obtained with both experimental positive and negative triangularity conditions.

\section{NONLINEAR SIMULATIONS}

We discuss in this Section the results of nonlinear simulations, looking at the simulated heat flow $\left\langle Q_{e}\right\rangle \cdot S$. Here $\left\langle Q_{j}\right\rangle=\int \mathbf{Q}_{j} \cdot \nabla x J^{x y z} d x d y d z / L_{x} L_{y} \int|\nabla x| J^{x y z} d z, J^{x y z}$ being the Jacobian of the $(x, y, z)$ coordinate system, while $S$ is the area of the flux surface of interest. $\mathbf{Q}_{j}$ is the $j-t h$ species heat flux, defined as $\mathbf{Q}_{j}=\int\left(1 / 2 m v^{2} f_{j} v_{\mathbf{E} \times \mathbf{B}}\right) d^{3} v$, where $f_{j}$ is the fluctuating part of the distribution function of the species $j$ and $v_{\mathbf{E} \times \mathbf{B}}$ the $\mathbf{E} \times \mathbf{B}$ velocity. It is compared to the experimental heat flow obtained from ASTRA interpretive simulations. Profiles in input to ASTRA have been varied within their error bars in order to obtain an estimate of the uncertainty on the power balance. 
GENE runs are carried out with a high level of realism, i.e. they are fully electromagnetic, collisional multispecies runs. Simulations are typically run up to 300-400 $\mathrm{a} / \mathrm{c}_{\mathrm{s}}$ in order to collect enough time statistics during the nonlinear saturated state. An estimate of the uncertainty associated to each run is obtained by computing a time averages over fixed time windows (typically of $\sim 50 a / c_{s}$ ) and then evaluating the variance of the obtained ensemble of mean values. In the following plots error bars should thus be interpreted as one standard deviation around the mean. We have verified that increasing by a factor of two the length of the time window used for averaging does not affect the results.

We remark that the electromagnetic flux, though accounted for, is as expected smaller by at least an order of magnitude compared to the electrostatic one. Also, the impurity heat flux is negligible, and therefore for clarity it will not be shown in the following plots. Finally, for the nominal plasma parameters we have also verified that accounting for the rotation as measured in the experiment does not significantly affect the simulated heat fluxes.

Aiming at understanding if the actual transport level can be obtained with local simulations within experimental error bars, for each plasma condition we have systematically varied the electron and ion temperature gradient lengths $\left(a / L_{T_{e}}\right.$ and $\left.a / L_{T_{i}}\right)$, as well as the electron density one $a / L_{n_{e}}$. In this latter case we have kept constant the impurity profile as measured in the experiment and adjusted the main ion logarithmic gradient in order to satisfy quasi-neutrality. Similar to linear runs, we estimate the experimental error bars in reconstructing temperature and density profiles to be approximately $\pm 20 \%$, and used this estimate to vary the values input to our simulations.

\section{A. Positive triangularity}

The results of simulations considering positive triangularity are summarized in Figure 6 where we plot, separately for electrons (red circles) and ions (blue squares), the simulated heat flows. For comparison the experimental values are marked with dashed lines of the same color.

Looking first at Phase I, for nominal parameters we observe an underestimation of the electron heat flux, by roughly $50 \%$, while the ion one is overestimated by $\sim 15 \%$. Contrary to linear simulations, the heat flux is mostly sensitive to $a / L_{n_{e}}$ but almost unaffected by variations of $a / L_{T_{e}}$. The latter has only a minor effect $(\sim 10 \%)$ on both transport channels, 
a)
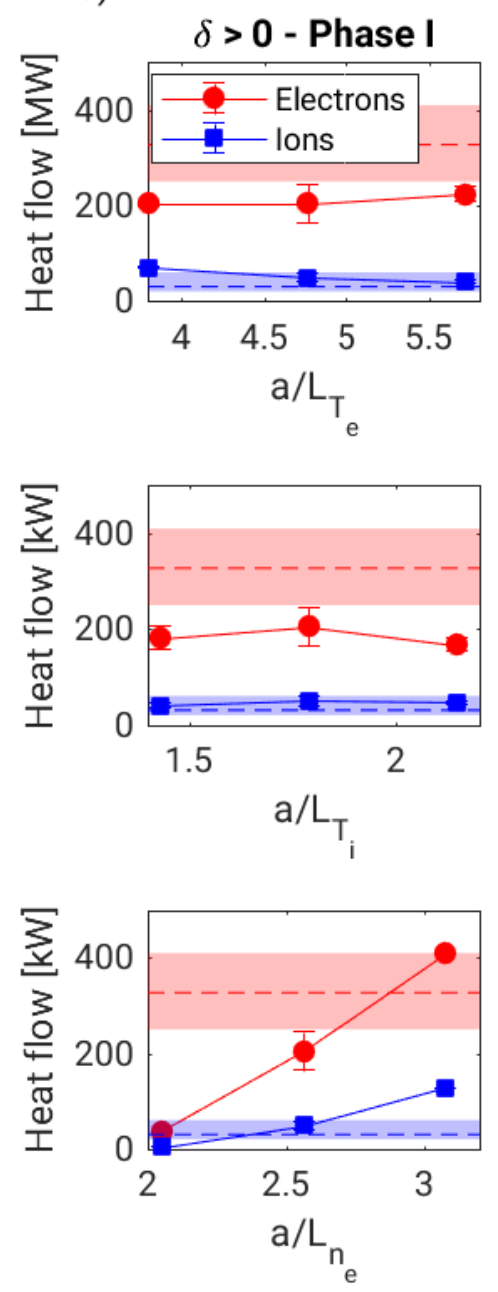

b)
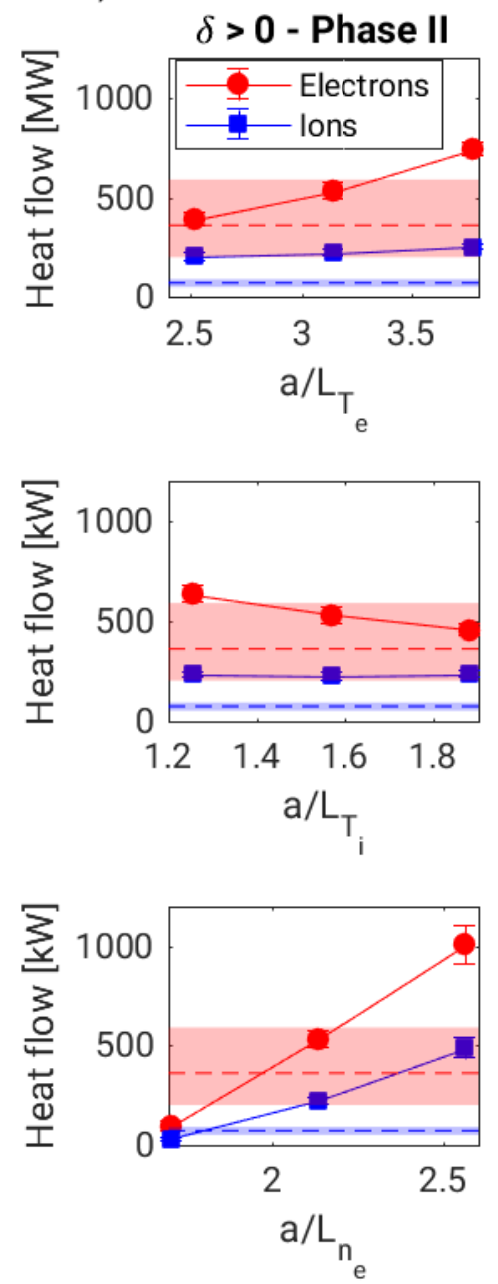

c)
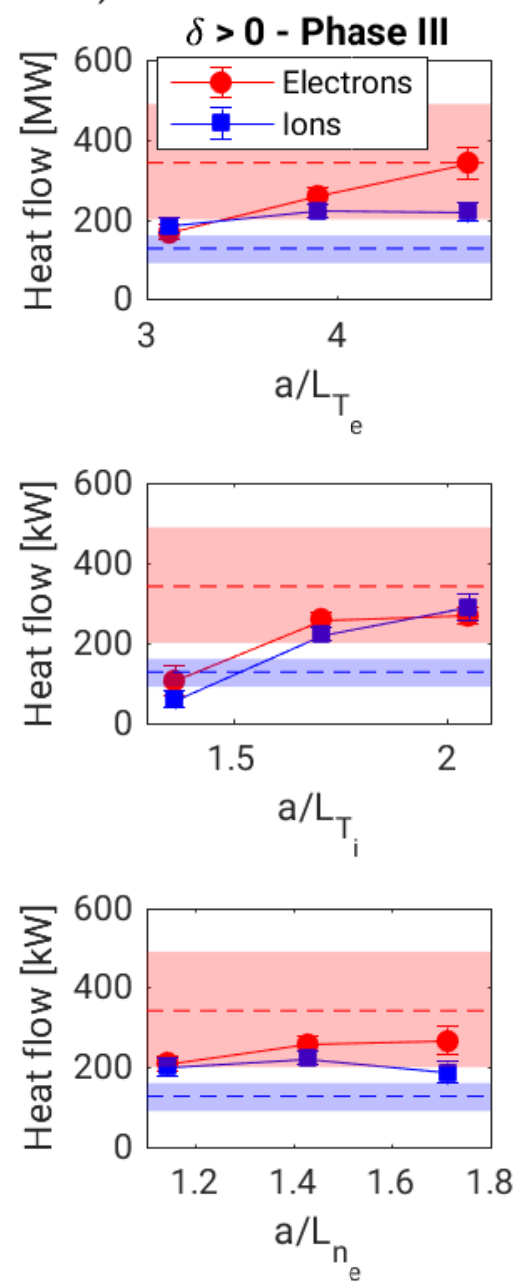

FIG. 6. Simulated heat flows in $\mathrm{kW}$ for TCV discharge \# $60797(\delta>0)$ and three different NBI powers: (a) $0 \mathrm{~kW}$ - Phase I, (b) $300 \mathrm{~kW}$ - Phase 2, and (c) $900 \mathrm{~kW}$ - Phase 3. For each phase heat flows are shown as a function of $a / L_{T_{e}}$ (first row), $a / L_{T_{i}}$ (second row) and $a / L_{n_{e}}$ (last row) with red circles for the electron channel and with blue squares for the ion one. Error bars indicate one standard deviation. Experimental heat flows, obtained from ASTRA runs, are shown with dashed lines while the shaded area indicate relative error bars.

in the same way variations of the ion temperature gradient are not causing any significant effect. We find that an increase of about $10 \%$ of the electron density gradient is sufficient to bring the simulated electron transport level to the experimental one.

When considering the results for Phase II, simulations for nominal parameters overestimate the transport level, for both channels by approximately 30\%. Differently from Phase 
I, the electron heat flow is almost equally sensitive to electron temperature and density gradients (as found in linear runs). We also observe that an increase in the ion temperature gradient, while leaving unaffected the ion heat flux, causes a non-negligible reduction of the electron one. Also in this scenario, even though simulations carried out with the nominal plasma parameters do not exactly reproduce the actual transport level, a $\sim 10 \%$ variation of the profiles is enough to reconcile simulations and experiments.

Finally, for Phase III the parameter affecting the most the electron transport level is the electron temperature gradient. Almost a $50 \%$ increase is found for a $20 \%$ increase of $a / L_{T_{e}}$ while only $\sim 10 \%$ for the same variation of $a / L_{n_{e}}$. In this case the heat flux is carried almost equivalently by ions and electrons, and while simulations recover the total transport level it appears more difficult to reconcile simulations and experiments for the two channels separately.

\section{B. Negative triangularity}

Nonlinear results for $\delta<0$ are summarized in Figure 7. Similarly to the positive triangularity case, the transport is dominated by the electron channel, carrying a flux larger by approximately a factor of two than the ion one. The heat flux is sensitive to both $a / L_{T_{e}}$ and $a / L_{n_{e}}$, with the latter being more effective when the beam is not turned on. Little or no effect is exerted by the main ion temperature gradient which, similarly to Phase II of Figure 6 , is found to lower $Q_{e}$ for the NBI heated plasma.

The major difference between $\delta>0$ and $\delta<0$ is the absolute magnitude of the transport level. For all $\delta<0$ cases considered here variations of the background profiles within error bars are not able to reproduce the actual transport level, which is over-estimated by at least a factor of 2, regardless of the NBI. Even though we have not carried out the corresponding simulation, one can speculate that for Phase I a combined reduction of $a / L_{T_{e}}$ and $a / L_{n_{e}}$ by $20 \%$ might be able to recover the experimental transport level. Those two parameters cause separately a reduction of respectively $25 \%$ and $50 \%$, assuming therefore that their effect is cumulative, they could cause a reduction from $\sim 1 \mathrm{MW}$ for nominal parameters to the experimental $184 \mathrm{~kW}$. The same combination of parameter changes, however, cannot be seen for Phase II.

A possible candidate to explain the transport reduction observed with negative triangu- 
a)
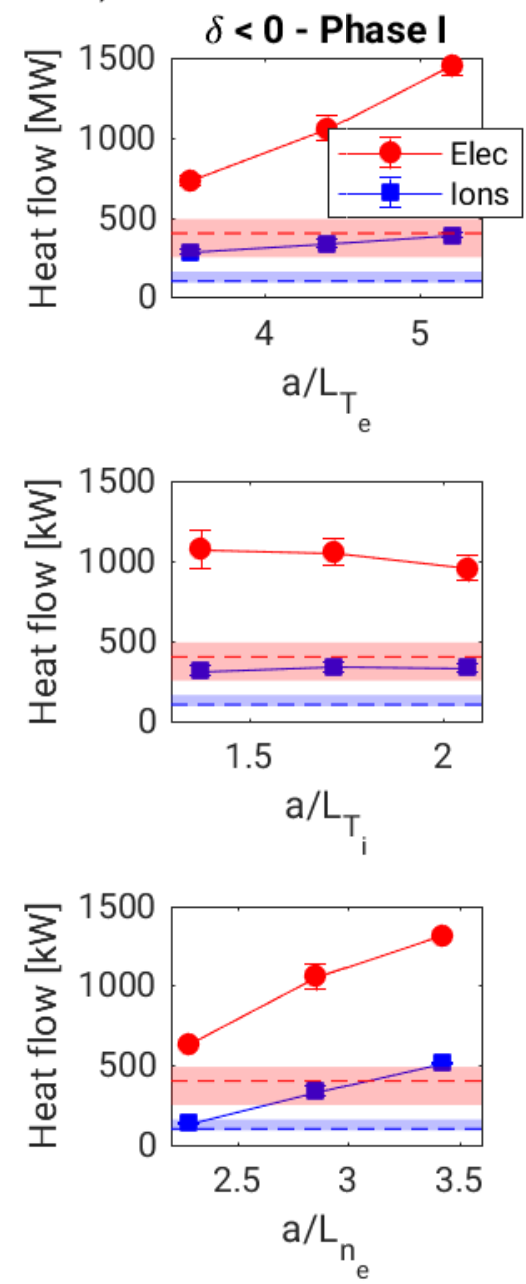

b)
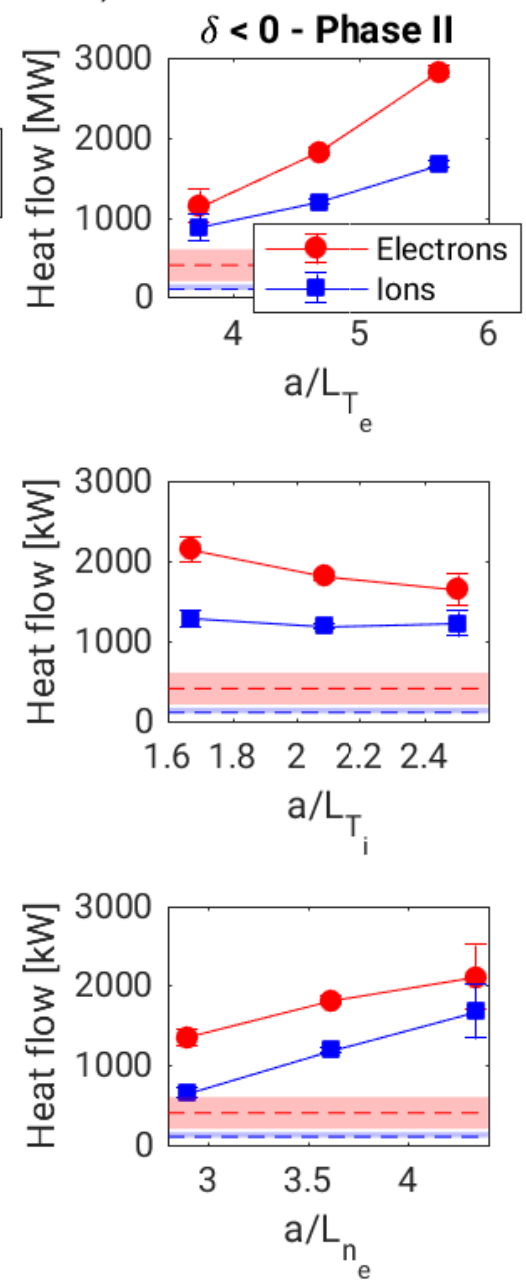

FIG. 7. Same as Figure 6 but showing results for negative triangularity.

larity is the impurity content, usually larger when $\delta<0$. Already in Ref. [3] it was noted how the effective collisionality $\nu^{*} \propto n_{e} Z_{\text {eff }} / T_{e}^{2}$ is the plasma parameter most effective in explaining the trends observed in experiments. Furthermore, it was shown in Ref. [6] how impurities are crucial in simulations to match the actual transport level, since an increase in $Z_{\text {eff }}$ causes a reduction of both ion and electron heat fluxes from dilution and collisional stabilization of TEMs. TCV is currently equipped with a Charge eXchange Recombination Spectroscopy (CXRS) system that measures the carbon content. A $\pm 20 \%$ error bar can be estimated for CXRS measurements [20] as well. Alternatively, one can evaluate a volume averaged $Z_{\text {eff }}$ based on an estimate of the neoclassical resistivity. The results of these two 


\begin{tabular}{|c|c|c|c|}
\hline & Phase I & Phase II & Phase III \\
\hline$\delta>0$, from CXRS & 1.19 & 1.22 & 1.15 \\
\hline$\delta>0$, from model & 1.52 & 1.66 & 1.48 \\
\hline \hline$\delta<0$, from CXRS & 1.48 & 1.36 & - \\
\hline$\delta<0$, from model & 2.61 & 2.97 & - \\
\hline
\end{tabular}

TABLE III. Value of $Z_{\text {eff }}$ evaluated from CXRS measurement and from a 1D model of plasma resistivity.

methods are summarized in Table III. A strong discrepancy is found for the cases with $\delta<0$, potentially affecting the transport level. Additional runs have thus been carried out to investigate the impact of $Z_{\text {eff }}$. We have considered first an increase of carbon concentration by $20 \%$ (while keeping constant $a / L_{c}$ ) and an increased impurity content such as to match the value of $Z_{\text {eff }}$ obtained from resistivity. In this latter case the same $a / L_{n}$ is considered for all species. The corresponding results are depicted in Fig. 8. As expected,
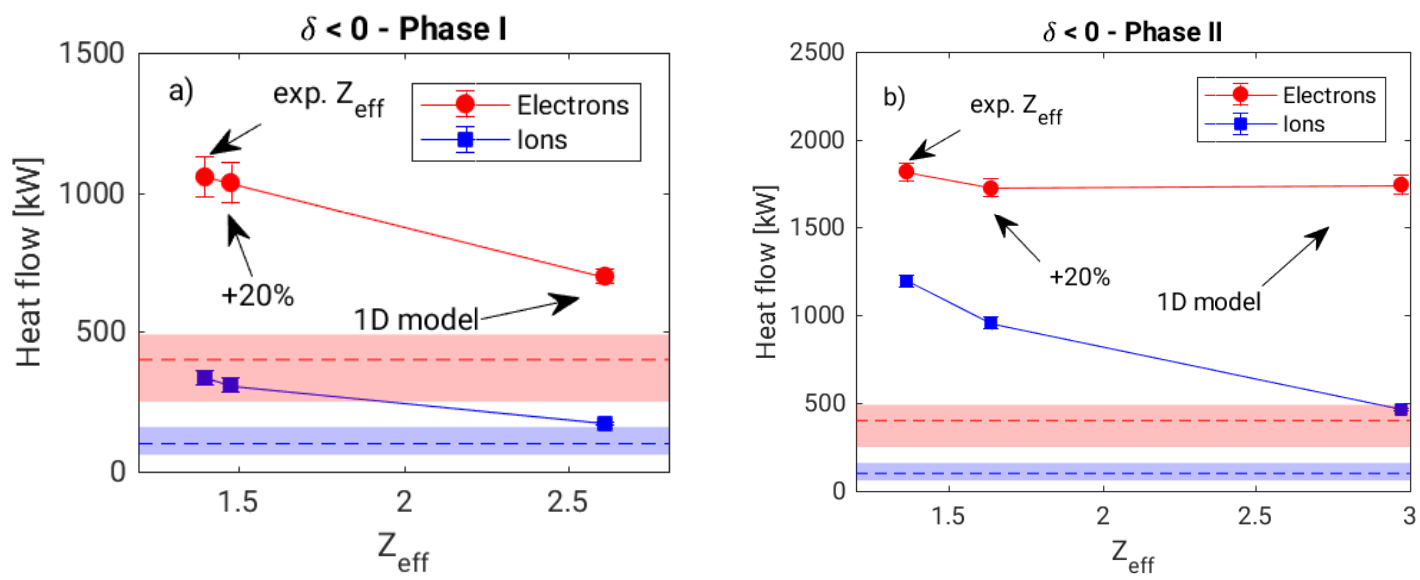

FIG. 8. Heat flow as a function of $Z_{\text {eff }}$ for the two phases of the negative triangularity plasma.

we obtain a reduction of the transport level when the impurity concentration is increased. This stabilization is stronger for parameters of Phase I, for which transport through both channels is reduced by roughly $40 \%$. An even stronger reduction is observed for the ion channel during Phase II, from $1.2 \mathrm{MW}$ to $460 \mathrm{~kW}$. The electron heat flux is however almost insensitive to the increase of the impurity content and remains off by a factor of three even for the highest $Z_{\text {eff }}$ considered.

The last parameter we modified aiming at matching the experimental transport level 
is the electron temperature. It enters in our simulations via three main contributions. $i$ ) It defines the plasma $\beta$ and the associated electromagnetic fluctuations, which are however negligible. $i$ i) It appears in the collision frequency where a reduction of $T_{e}$ causes an increase of collisionality and potentially a stabilization of TEMs. iii) It is used in the normalizations for converting GyroBohm to physical units, thus the same normalized transport level will correspond to an actual lower one if $T_{e}$ is lower. The results of this investigation are shown in Figure 9.
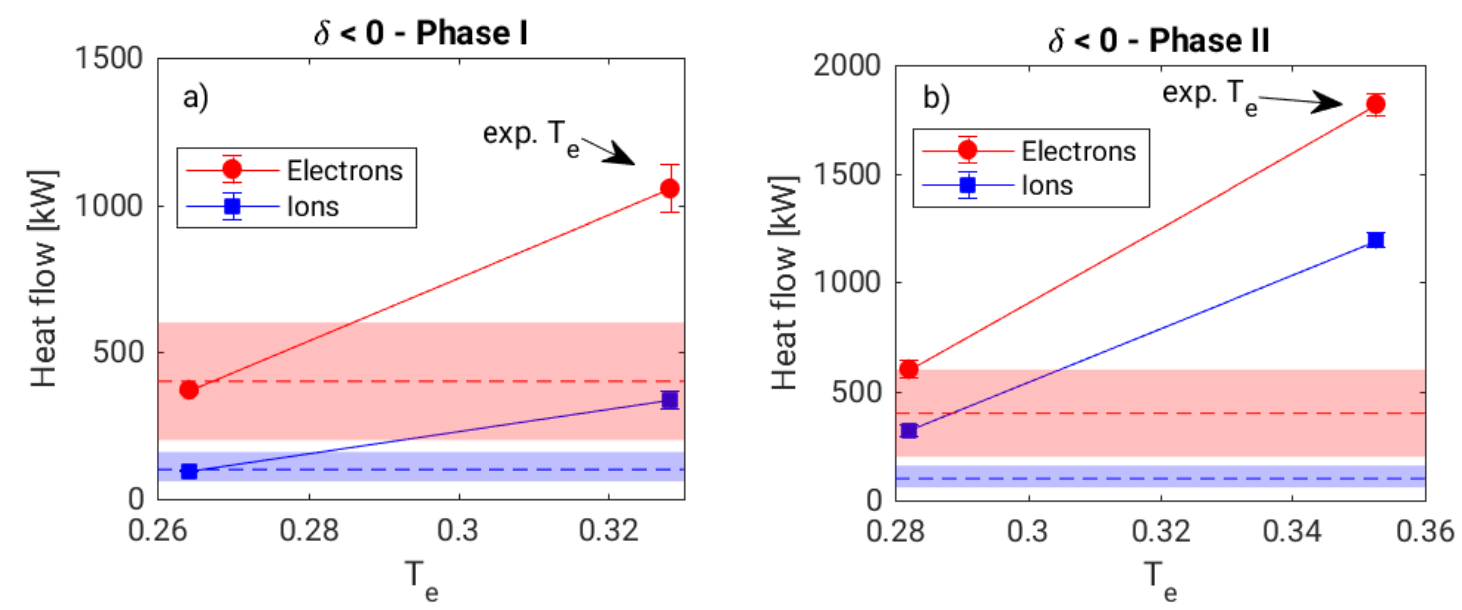

FIG. 9. Heat flow as a function of the electron temperature $T_{e}$, on the left for Phase I and on the right or Phase II of the $\delta<0$ plasma. Simulations have been performed considering the nominal value of $T_{e}$ and a $20 \%$ reduction. Experimental transport level is marked with a dashed line.

For the plasma parameters of Phase I we remark a surprisingly good agreement between simulations and measurements with the electron temperature reduction. A very strong reduction of transport is observed also for the parameters assumed for Phase II, even though in this case an overestimation still remains. This however is much smaller than what is obtained for nominal parameters and it now appears that minor changes of other parameters, most likely $a / L_{T_{e}}$ (see Fig. 7.b), are sufficient to recover the actual transport level from simulations. The transport reduction is a combined result of both larger collisionality and reduced GyroBhom units. The increase of collisionality reduces the electron flux by roughly 40\% (from $30.1 Q_{G B}$ to $18.5 Q_{G B}$ ) in Phase I and by $45 \%$ (from $40 Q_{G B}$ to $22 Q_{G B}$ ) for Phase II. Here $Q_{G B}=T_{e} n_{e} c_{s}\left(\rho_{s} / a\right)^{2}$. At the same time the variation of the GyroBohm units $Q_{G B}$ accounts for approximately $60 \%$ of the variation during both phases (going from 4.57 to 2.66 in Phase I and from 6.24 to 3.57 in Phase II). 


\section{AN IDEALIZED INVESTIGATION OF THE EFFECT OF TRIANGULARITY IN ITG DOMINATED PLASMAS}

From all the simulations presented in the previous sections, it appears clear how one can reproduce the measured transport level with variations of input parameters that are compatible with uncertainties in profile measurements and their reconstruction. While on the one hand this is clearly a positive conclusion of our investigation, on the other hand it also represents a limitation to our efforts at addressing the effect of $\delta$ on transport, for at least two different reasons. First, we need more constraints derived from experimental measurements in order to identify the plasma parameters that actually match the experiment. For each condition one can likely identify more than just one combination of parameters that gives the desired transport level, thus one would need detailed measurements of e.g. fluctuation amplitudes (of both temperature and density) in order to limit the possibilities. This kind of investigation can be carried out, but is computationally expensive and thus beyond the scope of the present work. Second, even if transport and fluctuations for discharges with positive and negative triangularity are reproduced, one still needs to be able to attribute any observed difference between the two to a change in triangularity (or potentially in few additional major parameters). This is usually very difficult to achieve in experiments (cf. differences in all parameters listed in Tables I and II). In addition, all the experimental conditions considered here are dominated by TEM modes, even for the highest heating power, which doesn't allow

us to reach any conclusion regarding the effect of $\delta$ in ITG dominated plasmas. Aiming at partially resolving these issues, we have designed and carried out a series of numerical experiments, which are discussed in this Section.

In order to isolate the effect of negative triangularity, we have carried out simulations using the GENE interface to the Miller geometry [21], which allows us to vary $\delta$ while keeping constant all other parameters. In this case, the contour of a given magnetic surface is given in cylindrical coordinates $(R, Z, \varphi)$ by:

$$
\begin{aligned}
& R=R_{\text {geom }}+r \cos \{\theta+\arcsin [\delta \sin \theta]\}, \\
& Z=Z_{\text {geom }}+\kappa r \sin [\theta+\zeta \sin (2 \theta)],
\end{aligned}
$$

where $R_{\text {geom }}=\left[R_{\max }+R_{\text {min }}\right] / 2$ is the geometric center of the flux surface, $R_{\max }$ and $R_{\min }$ being respectively its maximum and minimum major radius, $\theta$ is the poloidal angle and the 
elongation $\kappa$ and squareness $\zeta$ have been introduced.

We choose as reference the experimental conditions of Phase I and $\delta>0$. The values of the shaping parameters, together with their radial derivatives required to define all the metric coefficients necessary to set-up the corresponding GENE run, have been obtained from fitting the $\rho_{t o r}=0.685$ surface according to Eqs. 2 and 3. They are summarized in Table IV. We remark that the same edge elongation has usually a stronger penetration

\begin{tabular}{|c|c||c|c|}
\hline$\kappa$ & 1.29 & $d \kappa / d r$ & 0.19 \\
\hline$\delta$ & 0.19 & $d \delta / d r$ & 0.41 \\
\hline$\zeta$ & $0.8210^{-2}$ & $d \zeta / d r$ & 0.045 \\
\hline$\alpha_{\mathrm{MHD}}$ & 0.18 & $d R_{\text {geom }} / d r$ & -0.47 \\
\hline
\end{tabular}

TABLE IV. Parameters defining the flux-surface with $\delta>0$ used for carrying out the simulations based on Miller geometry.

depth when $\delta<0$ [22], an effect that we neglect here, but given the stabilizing effect of $\kappa$ on turbulence [23], this can be considered as a conservative approach. Similarly, we do not consider the different Shafranov shift that would be obtained constructing a global MHD equilibrium. Background gradients are chosen to match the experimental transport level, interpolating the results of Figure 6 .

Since the TCV experiments we have analyzed are dominated by TEMs/ETG, one needs to vary plasma parameters in order to access a regime where ITG are the most unstable modes. To this end, as shown in Figure 10 for the mode $k_{y} \rho_{s}=0.3$, we have varied the ion temperature as well as $a / L_{T_{i}}$. All other plasma parameters are kept constant. We indeed find that for each value of the ion temperature, a sufficiently large value of $a / L_{T_{i}}$ destabilizes ITG modes. We arbitrarily choose a point representative of this regime by increasing the ion temperature by $50 \%$ and doubling the experimental value of $a / L_{T_{i}}$, such as to be sufficiently far from the TEM-ITG transition.

The corresponding transport level obtained for both the experimental gradients and the ITG dominated case is plotted in Figure 11. We observe that for the experimental (TEM) case, the Miller parametrization reproduces almost perfectly the measured transport level (310 kW for the electron channel compared to $327 \mathrm{~kW}$ ), and a reduction of approximately a factor of three is observed in the transport level when going to negative triangularity (from 


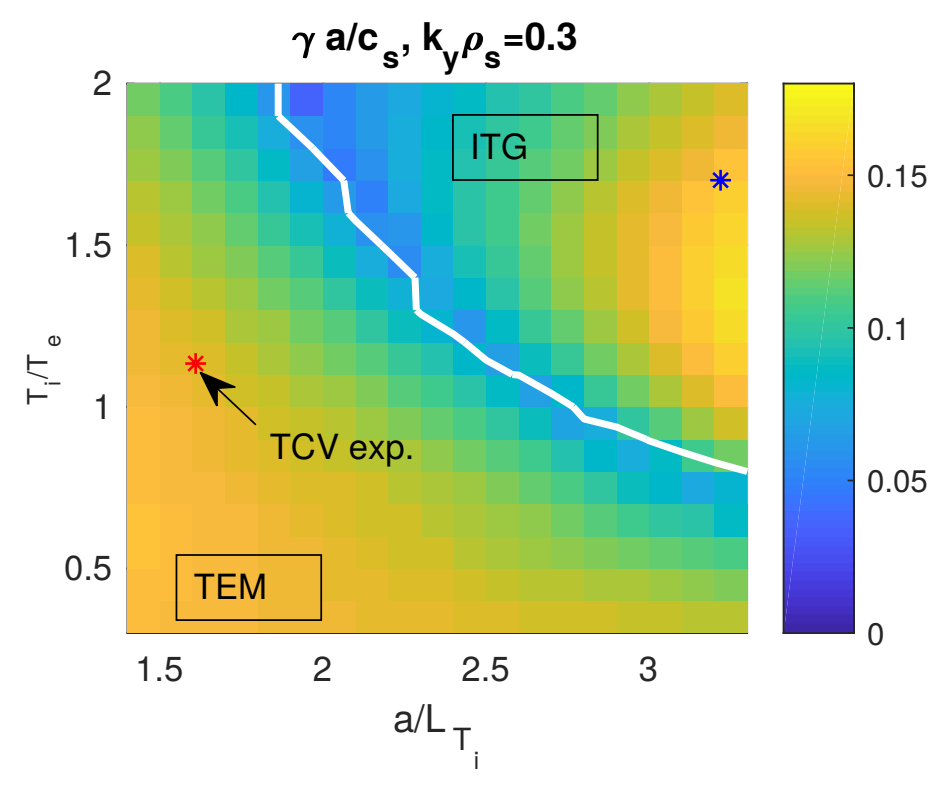

FIG. 10. Growth rate for the mode $k_{y} \rho_{s}=0.3$ as a function of the ion to electron temperature ratio $T_{i} / T_{e}$ and ion gradient length $a / L_{T_{i}}$. The plasma shape is computed according to the parameters listed in Table IV. The experimental TCV conditions are shown with a red star, while the one chosen as representative of an ITG regime with a blue star.

310 to $95 \mathrm{~kW}$ ). Similarly, a transport reduction is observed also in the ITG dominated case. In this case the transport level is, as expected, dominated by the ion channel (approximately larger by a factor of three with respect to the electron one) and is reduced by roughly $50 \%$ in both channels when the sign of triangularity is flipped. An explanation of the mechanism behind the transport reduction is beyond the scope of this work and will be the subject of a following publication. Nevertheless, in order to compare these results with previous work available in literature, we have looked at the distribution in velocity space of the heat flux in order to isolate any group of particles responsible for the transport, as summarized in Fig. 12. In the TEM dominated regime (Figure 12.a) the reduction happens mostly for a reduction of the heat flux carried by trapped particles, by a factor of 5.7 or the electrons and $\sim 5$ for the main ions. The heat flux carried by passing particles is instead reduced by about a factor of three for both species. These trends are in agreement with what reported in Ref. [5], we cannot however claim them to be universal since we have considered only one specific plasma condition.

In the ITG dominated case instead, summarized in Figure 12.b, the reduction is uniform 

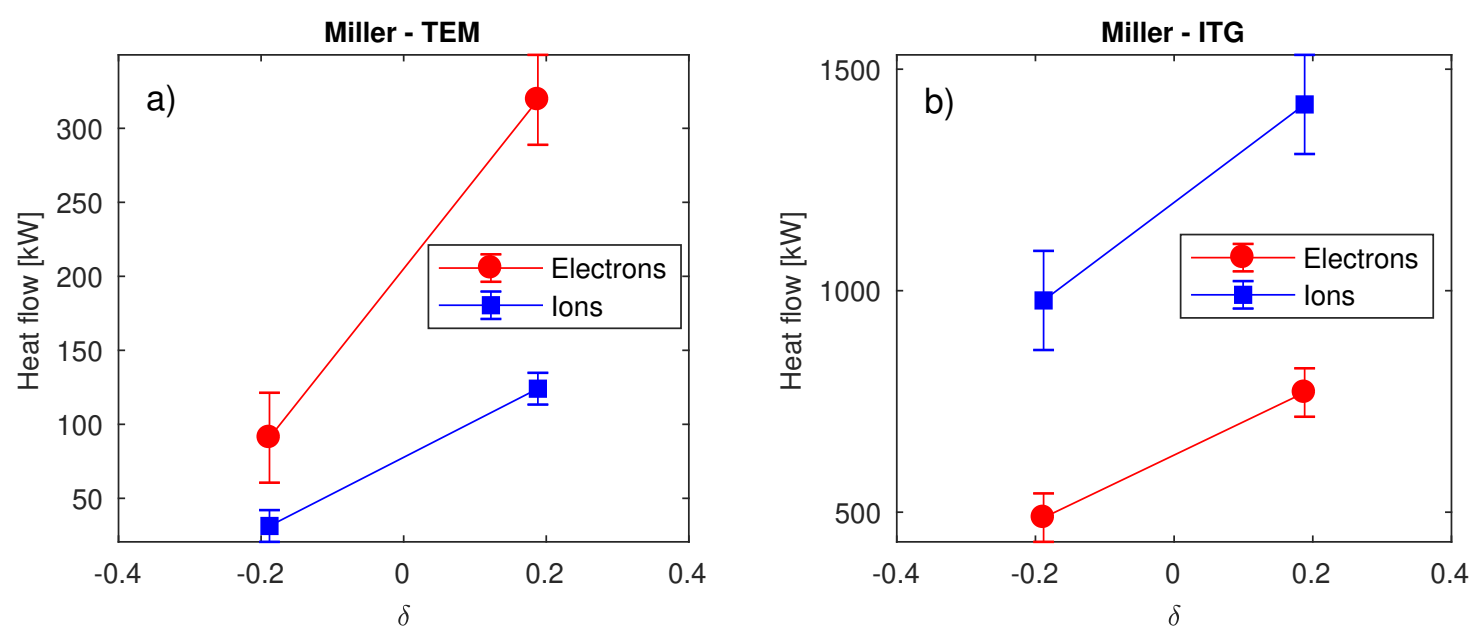

FIG. 11. Transport level obtained considering plasmas with Miller geometry and varying only the sign of triangularity. Shown are the heat flows as a function of triangularity, for (a) TEM regime based on the actual measured TCV profiles and (b) an ad-hoc profiles in the ITG regime. Electron heat flow is shown with red circles while ion in blue squares.
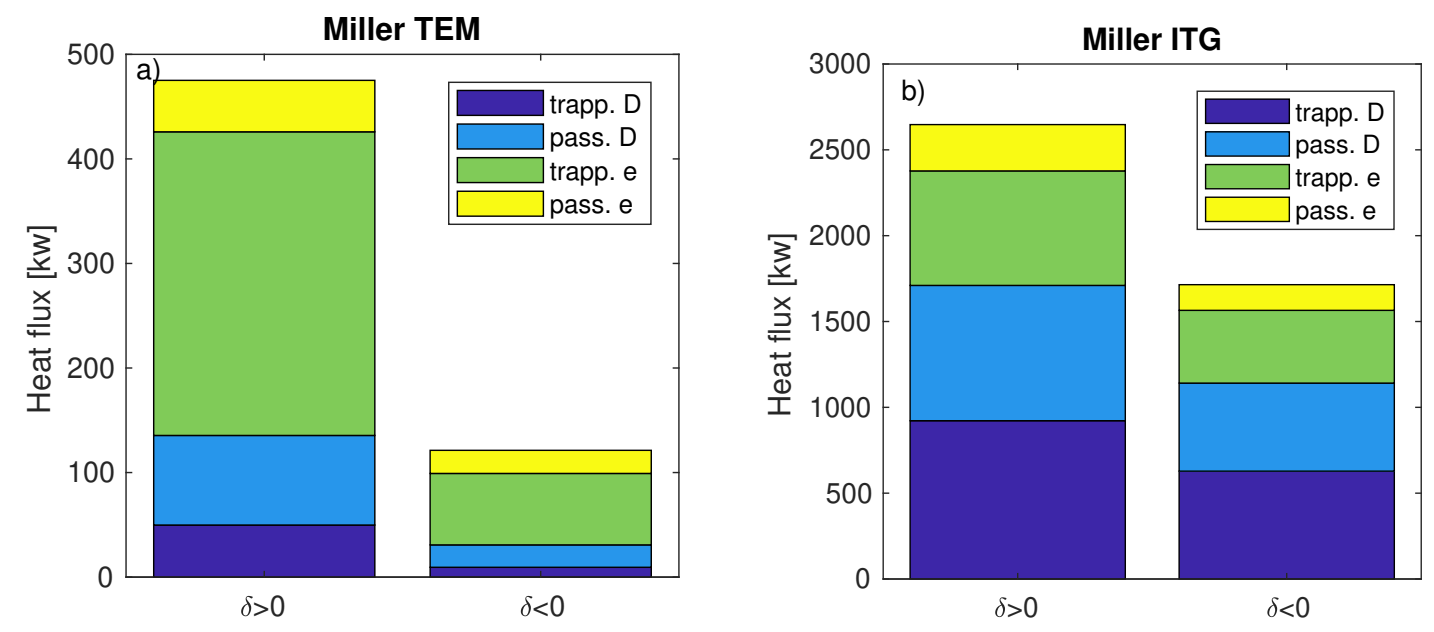

FIG. 12. Distribution of the heat flux between trapped and passing particle for (a) TEM and (b) ITG cases.

among trapped and passing channels for both species. In both positive and negative $\delta$ the ion transport is shared almost equivalently between trapped and passing channels (constituting respectively $55 \%$ and $45 \%$ of the total), while trapped electrons carry about $75 \%$ of the electron heat flux in both shapes. 


\section{CONCLUSIONS}

We have carried out a series of local (flux-tube) gyrokinetic simulations looking at the effect of negative triangularity on turbulent transport and its interplay with other plasma parameters, mainly the ion to electron temperature ratio $T_{i} / T_{e}$. To this end, we have considered realistic plasma conditions measured in the TCV tokamak.

The main finding of our investigation can be summarized as follows:

(i) For all plasma conditions considered, the leading instabilities are TEMs and ETG. Despite the different external heating power (up to $1 \mathrm{MW}$ for $\delta>0$ ) ITGs are not observed within experimental error bars at the position we have analyzed. Increasing the temperature ratio with NBI heating is in fact found to destabilize ETG-like modes.

(ii) Local gyrokinetic simulations are able to reproduce the measured transport level if profiles are adjusted with variation compatible with the experimental error bars.

(iii) Matching the flux appears to be easier for $\delta>0$ than for $\delta<0$. In this latter case one needs to reduce the electron temperature as well (see Fig. 9).

(iv) Linear simulations show that the plasma is dominated by trapped electron modes, driven by both density and temperature gradients. Little or no effect is played for the considered parameters by ion-to-electron temperature ratio and ion temperature gradient.

(v) Extensive nonlinear scans of the turbulence-driving gradients have been performed, for both plasma shapes and different NBI heating powers (Figures 6 and 7). One observes a stronger sensitivity to $a / L_{n_{e}}$ for Ohmic conditions and to $a / L_{T_{e}}$ for NBI heated plasmas. The main ion temperature gradient does not affect transport in Ohmic scenarios, while it lowers the the electron heat flow when the NBI is turned on.

(vi) Accounting for the uncertainty in the electron temperature gradient is a crucial element to bring simulation results and experiments in agreement. Lowering $T_{e}$ within error bars strongly reduces the flux because of both increased collisionality and change in the GyroBohm units.

(vii) The effect of impurity content, one of the plasma parameters for which experimental uncertainty is largest, has been investigated for $\delta<0$. TCV measurements relative 
to negative triangularity have up to $100 \%$ uncertainty (Table III). Increasing the $\mathrm{C}$ impurity content does cause a flux reduction, alone it is however not enough to recover the experimental transport level.

(viii) In order to investigate the effect of negative triangularity on ITG dominated discharges, we have carried out a numerical experiment in which we have chosen the value of $a / L_{T_{i}}$ and $T_{e} / T_{i}$ such that the most unstable modes at the ions scale are ITGs. In order to isolate the effect of $\delta$ from other parameters the magnetic geometry is prescribed using Miller's model, based on the actual TCV equilibrium. ITG dominated plasmas show the same transport reduction for both ion and electron channels as found experimentally and in simulations for TEM dominated regimes.

(ix) While in the TEM dominated regime the reduction is mostly due to a reduction via the trapped particles channel, in the ITG regime a uniform reduction among all channels and species is observed.

The simulations presented in this work represent a systematic investigation of the effect of $\delta$ on the transport level and predict a transport reduction for negative triangularity plasmas in the ITG regime. Further investigations are however required in order to fully explore the effect of $\delta$ on ITGs and possibly explain the physical mechanism behind these observations. Of particular relevance are the complementary observations reported from the DIII-D tokamak. An investigation similar to the one discussed here for TCV conditions would be particularly interesting and allow one to confirm or disprove trends and potentially identify other stabilizing mechanisms. Finally, an aspect that needs further investigation is the importance of global effects. While often invoked as the crucial ingredient necessary to reproduce the actual transport level for machines of the size of TCV, our results show that, at least for the conditions we have considered, this may not be the case. Nevertheless, even if a local description captures the actual heat fluxes, nonlocal effects can still play a relevant role causing e.g. a different radial penetration of the stabilizing effect of $\delta<0$ depending on shape. This possibility cannot be ruled out without carrying out the corresponding global simulations. This investigation is however left for future work, because such runs are computationally significantly more expensive and it is crucial to use inputs with an uncertainty as low as possible. 


\section{ACKNOWLEDGMENTS}

We thank C. Marini and F. Carpanese for helping with ion profile fitting as well as for very fruitful discussions regarding the CXRS diagnostic and equilibrium reconstruction. Useful discussion with S. Brunner are greatly acknowledged. This work was supported in part by the Swiss National Science Foundation and by a grant from the Swiss National Supercomputing Centre (CSCS) under projects ID s793 and s863. Part of the computations were carried out using the Marconi-FUSION supercomputer system at the CINECA, Italy. We acknowledge PRACE for awarding us access to MareNostrum at Barcelona Supercomputing Center (BSC), Spain.

\section{Appendix A: Profile fitting and associated error bars}

Electron temperature and density profiles are obtained from Thomsom scattering measurements, while the main ion values are inferred from Charge eXchange Recombination Spectroscopy (CXRS) diagnostic in TCV [24]. The same temperature of the main ions and carbon is considered. The data is fitted using cubic splines with a tension that is adjusted in order to avoid over-fitting. A time interval of $12 \mathrm{~ms}$ is used to average the experimental data. A spline fit of the error bar is also performed in order to estimate the uncertainty of the fit itself. These values allow one to propagate the uncertainty on the gradients. The resulting profiles are shown in Figures 13 and 14, where we plot the experimental data (full markers), fit and corresponding error bar. A particularly large value of $T_{i} / T_{e}$ is observed for Phase III

at the edge. Besides systematic errors, a possible explanation of this can be attributed to the interaction between the heating neutral beam (NBH) and the diagnostic beam (DBNI) making more difficult to obtain high quality measurements at the edge. Based on experience in analyzing and fitting ion profiles in TCV, we do not expected that this affects profiles inside $\rho_{\text {tor }}=0.85$.

Error bars (one $\sigma$ ) computed following this procedure are typically $\leq 10 \%$ for electron profiles and about $10-15 \%$ for corresponding gradients; such an estimate however does not include systematic errors. As can clearly be seen from Figures 13 and 14, ion profiles have somewhat larger error bars than electron measurements, in particular for Phase III. Therefore, in order to account for possible systematic errors and to simplify the analysis, 

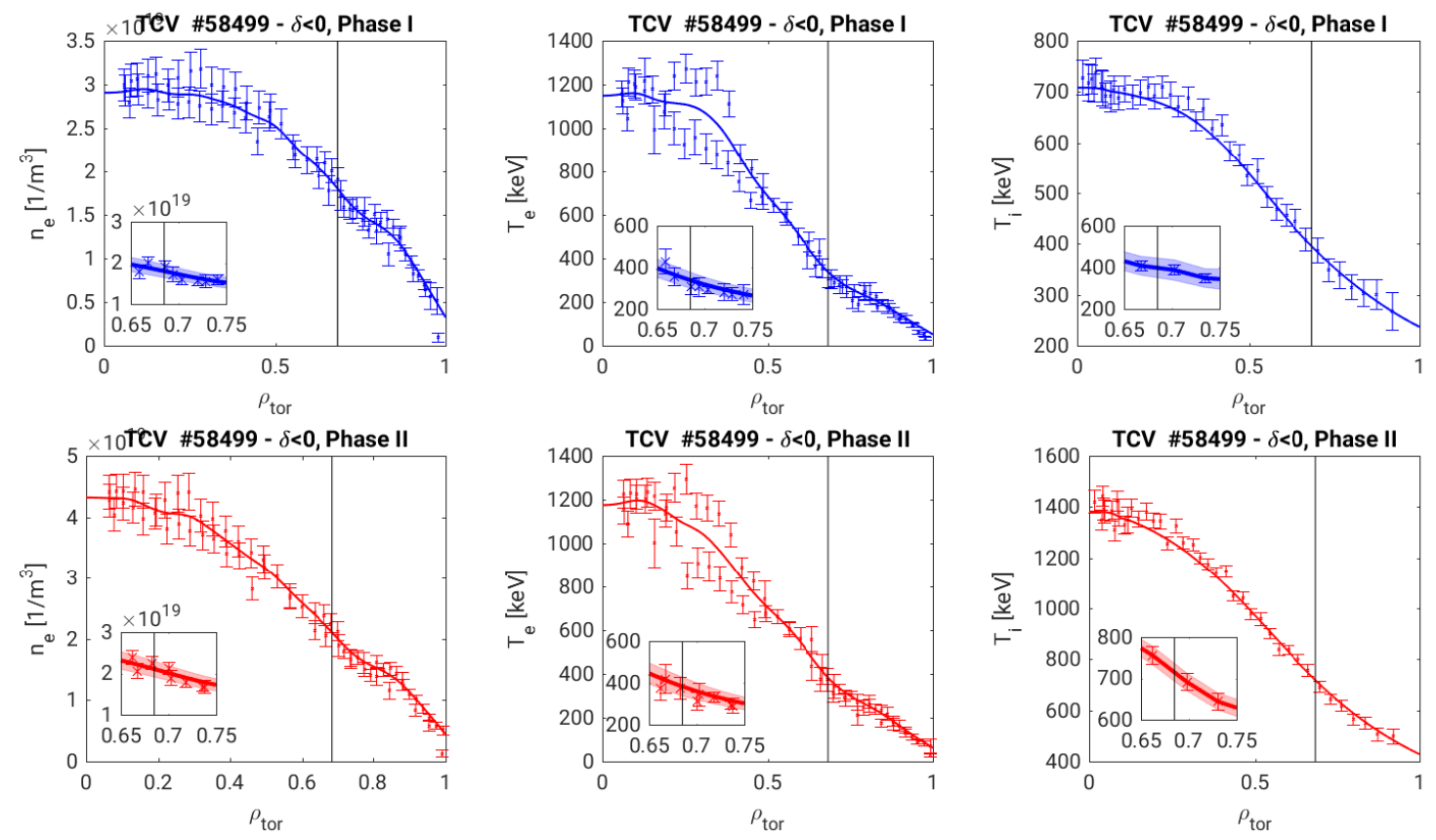

FIG. 13. From left to right, profiles of electron density, electron temperature and main ion for TCV shot \#58499 for Phase I (first row) and Phase II (second row). Solid markers indicate the experimental measurements together with $1 \sigma$ error bar, while to solid line the data fit. The radial location considered for simulations is marked with a vertical line. The inset shows the same data together with $1 \sigma$ statistical error bar of the fit.

we have increased the error bar estimate to $20 \%$ and considered it to be the same for all profiles.

\section{Appendix B: Linear sensitivity analysis}

A sensitivity analysis of the linear growth rate with respect to variations of the input profiles has been carried out and is presented in this Section. For all plasma conditions listed in Tables I and II we have varied the main turbulence drives in order to asses both their impact of the growth rates and identify any possible change in turbulence regime (namely a TEM to ITG transition). We have carried out this analysis for the mode $k_{y} \rho_{s}=0.3$, where we expect most of the nonlinear transport to occur. We have varied separately $a / L_{T_{e}}$ and $a / L_{n_{e}}$, the two major destabilizing parameters for TEM modes, and $a / L_{T_{e}}$ and $T_{i} / T_{e}$ in order to identify possible phase space regions dominated by ITG modes. When varying electron 

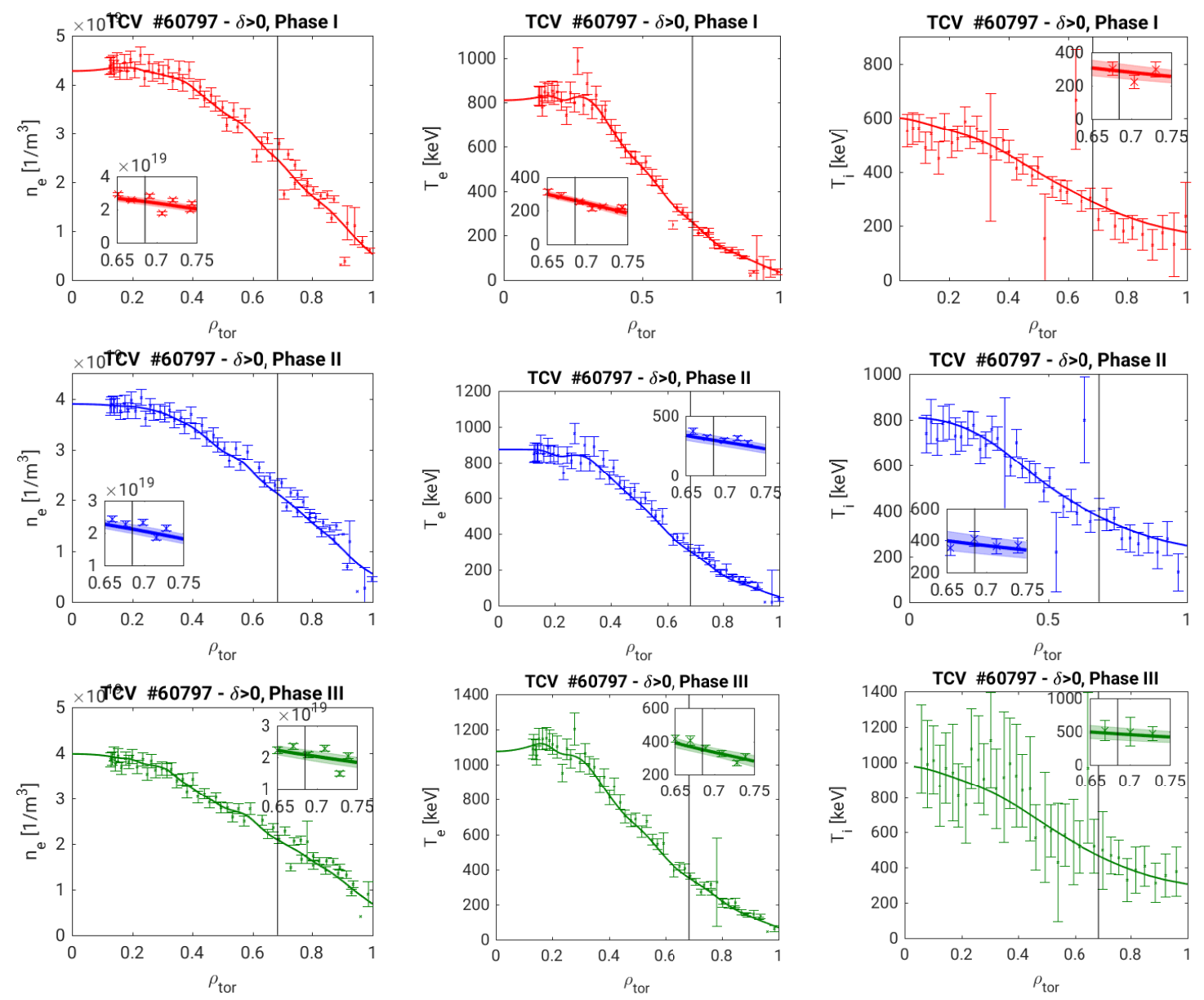

FIG. 14. Same as Fig. 13 but showing results for TCV discharge \#60797.

profiles we have kept the carbon values fixed, thus adjusting the main ion dilution to ensure quasi-neutrality. Given the particularly large error bars associated to all measurements, estimated as detailed in Appendix $\mathrm{A}$ in $\pm 20 \%$, and the relatively reduced computational cost of linear simulations, we have carried out this sensitivity analysis considering a larger $( \pm 30 \%)$ window of values, with the aim of providing a more complete analysis.

We observe that in all cases considered the most unstable mode has a negative frequency, i.e. it can be identified as a trapped electron mode. The only exception is Phase III where we observe that a transition to positive frequency can be obtained if the electron temperature gradient is reduced by $20 \%$ or $a / L_{T_{i}}$ is increased by $20 \%$. In all cases, except Phase II for $\delta>0$, the main drive for the TEM is the temperature gradient (in Phase I of positive triangularity the mode is equally sensitive to both $a / L_{T_{e}}$ and $\left.a / L_{n_{e}}\right)$. Linear growth rates are much less sensitive to variation of ion temperature gradient and ion to electron temperature 

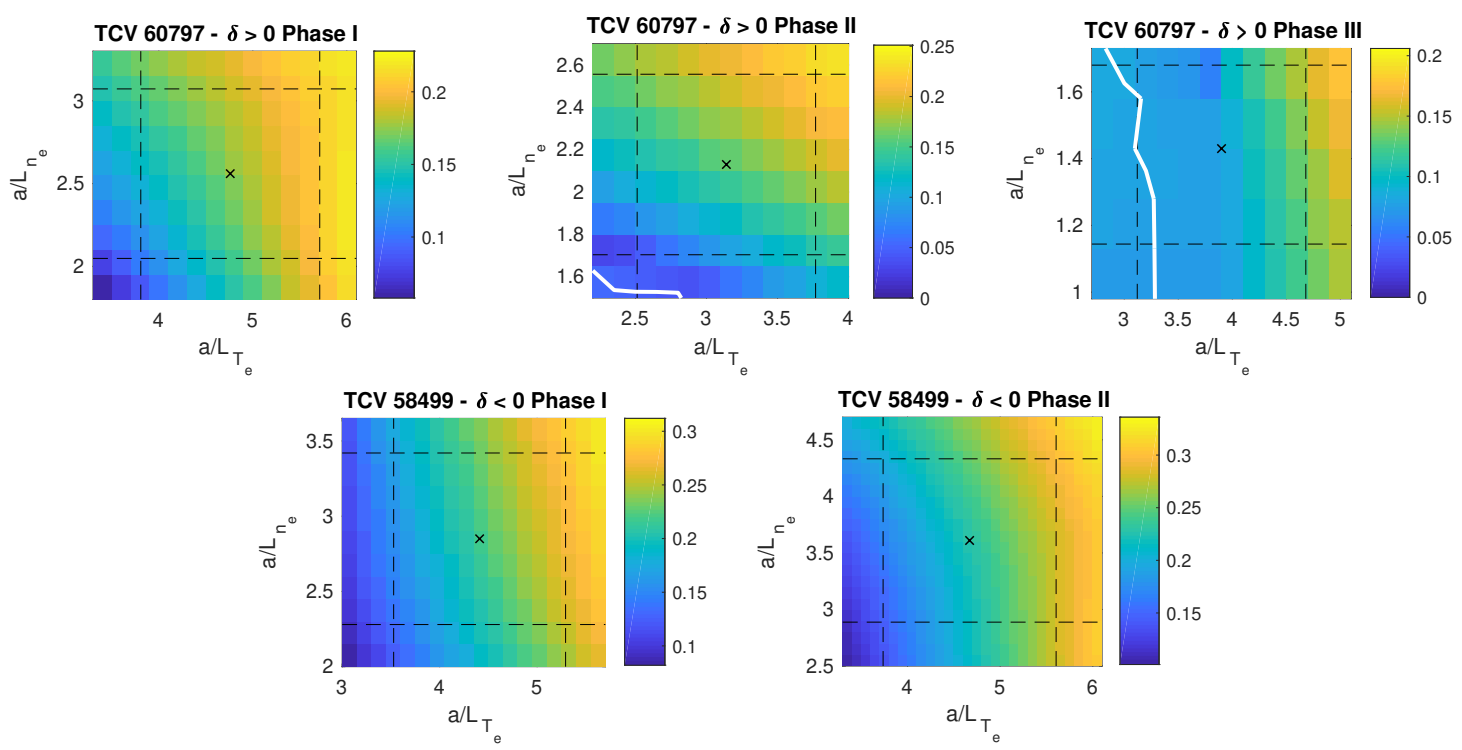

FIG. 15. Growth rate of the mode $k_{y} \rho_{s}$ as a function of $a / L_{T_{e}}$ and $a / L_{n_{e}}$ for the different phases considered. The nominal conditions are indicated with a black cross, while $\pm 20 \%$ error bars are delimited with dashed lines. White lines indicate a change in the real frequency.
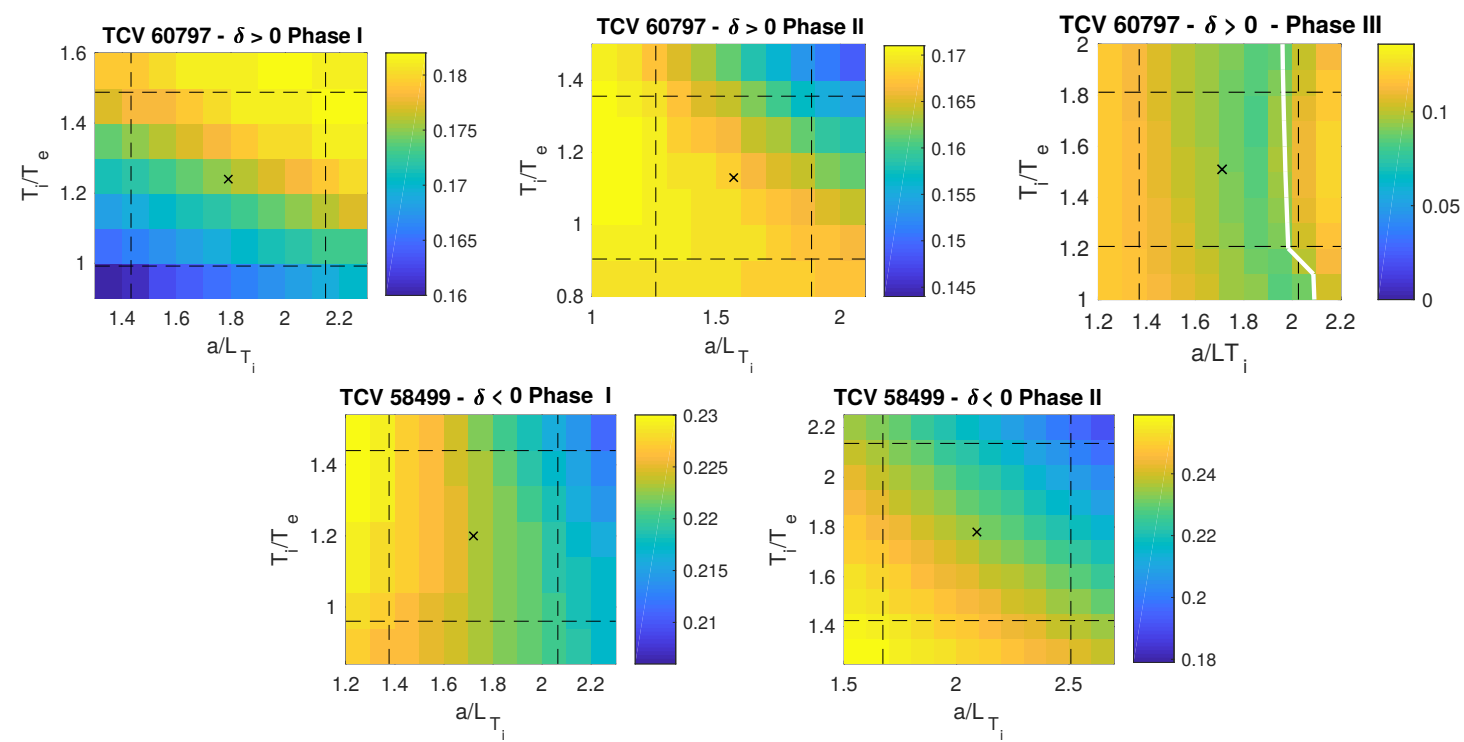

FIG. 16. Same as Figure 15 but showing the influence of $a / L_{T_{i}}$ and $T_{i} / T_{e}$.

ratio, as can be see in Fig. 16. 
[1] Fontana M, Porte L, Coda S and Sauter O 2017 Nuclear Fusion 58024002

[2] Huang Z and and S C 2018 Plasma Physics and Controlled Fusion 61014021

[3] Camenen Y, Pochelon A, Behn R, Bottino A, Bortolon A, Coda S, Karpushov A, Sauter O, Zhuang G and the TCV team 2007 Nuclear Fusion 47510

[4] Camenen Y, Pochelon A, Bottino A, Coda S, Ryter F, Sauter O, Behn R, Goodman T P, Henderson M A, Karpushov A, Porte L and Zhuang G 2005 Plasma Physics and Controlled Fusion 471971

[5] Marinoni A, Brunner S, Camenen Y, Coda S, Graves J P, Lapillonne X, Pochelon A, Sauter O and Villard L 2009 Plasma Physics and Controlled Fusion 51055016

[6] Merlo G, Brunner S, Sauter O, Camenen Y, Görler T, Jenko F, Marinoni A, Told D and Villard L 2015 Plasma Physics and Controlled Fusion 57054010

[7] Medvedev S, Kikuchi M, Villard L, Takizuka T, Diamond P, Zushi H, Nagasaki K, Duan X, Wu Y, Ivanov A, Martynov A, Poshekhonov Y, Fasoli A and Sauter O 2015 Nuclear Fusion 55063013

[8] Austin M E, Marinoni A, Walker M L, Brookman M W, deGrassie J S, Hyatt A W, McKee G R, Petty C C, Rhodes T L, Smith S P, Sung C, Thome K E and Turnbull A D 2019 Phys. Rev. Lett. 122(11) 115001

[9] Kikuchi M, Takizuka T and Furukawa M Negative Triangularity as a Possible Tokamak Scenario in Proceedings of the 12th Asia Pacific Physics Conference (APPC12)

[10] Medvedev S, Kikuchi M, Villard L, Takizuka T, Diamond P, Zushi H, Nagasaki K, Duan X, Wu Y, Ivanov A, Martynov A, Poshekhonov Y, Fasoli A and Sauter O 2015 Nuclear Fusion 55063013

[11] Marinoni A 2009 Plasma fluctuation studies in the TCV tokamak Ph.D. thesis EPFL Lausanne

[12] Karpushov A N, Chavan R, Coda S, Davydenko V I, Dolizy F, Dranitchnikov A N, Duval B P, Ivanov A A, Fasel D, Fasoli A, Kolmogorov V V, Lavanchy P, Llobet X, Marlétaz B, Marmillod P, Martin Y, Merle A, Perez A, Sauter O, Siravo U, Shikhovtsev I V, Sorokin A V and Toussaint M 2017 Fusion Engineering and Design 123468 - 472 ISSN 0920-3796 proceedings of the 29th Symposium on Fusion Technology (SOFT-29) Prague, Czech Republic, September 5-9, 2016 
[13] Fontana M, Porte L, Coda S, Sauter O, Brunner S, J A C, Merlo G and the TCV team 2019 submitted

[14] Fontana M 2018 Turbulence studies in TCV using the Correlation ECE diagnostic Ph.D. thesis EPFL Lausanne

[15] Angioni C, Peeters A G, Pereverzev G V, Ryter F and Tardini G 2003 Physics of Plasmas 10 $3225-3239$

[16] Moret J M, Duval B, Le H, Coda S, Felici F and Reimerdes H 2015 Fusion Engineering and Design 91 1-15

[17] Lütjens H, Bondeson A and Sauter O 1996 Computer Physics Communications 97219 - 260

[18] Jenko F, Dorland W, Kotschenreuther M and Rogers B N 2000 Physics of Plasmas 7

[19] Merz F 2008 Gyrokinetic simulation of multimode plasma turbulence Ph.D. thesis Westfälische Wilhelms-Universität Münster, Germany

[20] Marini C private communication

[21] Candy J 2009 Plasma Physics and Controlled Fusion 51105009

[22] Graves J P 2013 Plasma Physics and Controlled Fusion 55074009

[23] Angelino P, Garbet X, Villard L, Bottino A, Jolliet S, Ghendrih P, Grandgirard V, McMillan B F, Sarazin Y, Dif-Pradalier G and Tran T M 2009 Phys. Rev. Lett. 102(19) 195002

[24] Marini C 2017 Poloidal CX visible light plasma rotation diagnostics in TCV Ph.D. thesis EPFL Lausanne 